\title{
A Search for Host Galaxies of 24 Gamma Ray Bursts
}

\author{
J.-E. Ovaldsen ${ }^{1}$, A. O. Jaunsen ${ }^{1}$, J. P. U. Fynbo ${ }^{2}$, J. Hjorth ${ }^{2}$, C. C. Thöne ${ }^{2}$, C. Féron ${ }^{2}$, \\ D. $\mathrm{Xu}^{2}$, J. H. Selj ${ }^{1}$, J. Teuber ${ }^{1,2}$ \\ j.e.ovaldsen@astro.uio.no
}

\begin{abstract}
We report the results from observations of 24 gamma ray burst (GRB) fields from 2005 and 2006 undertaken at the Danish 1.54m telescope at ESO/La Silla. Photometry and positions for two previously unpublished host galaxy candidates (GRBs 050915 and 051021) are presented, as well as for eight other detected objects which are either known GRB hosts or candidate hosts. The candidates are suitable for spectroscopic follow-up in order to have their redshifts and other physical characteristics determined. In the cases where no likely host candidate is detected inside the refined Swift XRT error circle we are still able to put interesting and rather deep limits on the host magnitude. Based on our detections and upper limits we have performed simulations which suggest that the host galaxies are drawn from a fainter sample than previous (i.e. pre-Swift) studies.
\end{abstract}

Subject headings: gamma rays: bursts - cosmology: observations — galaxies: photometry — methods: data analysis

\section{Introduction}

The properties of galaxies hosting long gamma ray bursts (GRBs) were early on realized to give important clues on the nature of GRBs (Fenimore et al. 1993; Band \& Hartmann 1998; Hogg \& Fruchter 1999). Furthermore, it was also realized early on - in the socalled "afterglow" era, when the cosmological distance scale for long GRBs was established - that GRBs may be ideal tracers of (massive) star-formation throughout the observable Universe. Hence, a well selected

\footnotetext{
${ }^{1}$ Institute of Theoretical Astrophysics, University of Oslo, P. O. Box 1029, Blindern, N-0315 Oslo, Norway

${ }^{2}$ Dark Cosmology Centre, Niels Bohr Institute, University of Copenhagen, Juliane Maries Vej, DK2100 Copenhagen $\varnothing$, Denmark
}

sample of GRB host galaxies could be used to characterize the properties of the various types of galaxies that dominate the cosmic star-formation as a function of cosmic epoch (Totani 1997; Wiiers et al. 1998; Mao \& Mo 1998; Blain \& Natarajan 2000).

Prior to the currently very successful Swift satellite (Gehrels et al. 2004) there were a number of published host studies (e.g., Hogg \& Fruchter 1999; Le Floc'h et al. 2003; Christensen et al. 2004; Fruchter et al. 2006). The main conclusion based on these and other pre-Swift studies were that the hosts of GRBs are typically faint, star-forming dwarf galaxies. The median magnitude of pre-Swift hosts is about $R=25$. However, these conclusions are based on incomplete samples as typically only about $30 \%$ of pre-Swift GRBs are sufficiently well localized to allow an unam- 
biguous host identification (e.g., Fynbo et al. 2001; Berger et al. 2002). Therefore, these conclusions may not be valid for GRB hosts in general.

With the Swift satellite the rate of well localized long GRBs has gone up by about an order of magnitude. Furthermore, given the very rapid and precise localization, thanks primarily to the X-ray telescope (XRT) on Swift, the fraction of Swift GRBs without sub-arcsecond localization is now significantly smaller, and even based on the (refined) XRT positions alone it is possible to make interesting conclusions on the properties of the hosts.

So far no systematic study of the hosts of Swift GRBs has been published. We here make a first attempt to characterize a sample of hosts related to Swift GRBs based on relatively deep images collected at the Danish $1.54 \mathrm{~m}$ telescope on La Silla in Chile. The paper is organized as follows: We describe the observations and data set in Section 2 and the photometric reductions in Section $[3$. The results for our 24 GRB fields (22 Swift and two HETE bursts) are presented in Section 4, we derive upper limits for the brightness of the host for each GRB and provide positions and magnitudes for the detected host galaxy candidates. In Section 5 our photometric results for a subsample of 15 GRB fields - i.e. long Swift GRBs with confirmed optical transients (OTs) - are compared to the pre-Swift host sample of Fruchter et al. (2006). Our results give tentative evidence that GRB host galaxies from the post-Swift era are fainter than those belonging to earlier host studies. Section 6 summarizes our main findings.

\section{Observations and data set}

The data were obtained with the Danish $1.54 \mathrm{~m}$ telescope at the European Southern Observatory on La Silla, Chile, 2005 October 24 to November 21 and 2006 February 19 to
March 15. The CCD detector on the DFOSC (Danish Faint Object Spectrograph and Camera) instrument has a current default readout area of $2148 \times 2102$ pixels, a pixel scale of 0.395 arcsec pix $^{-1}$, a gain of 0.74 electrons $\mathrm{ADU}^{-1}$, and a readout noise of 3.1 electrons.

We selected our GRB targets (Table 1) mainly based on observability at the two epochs of observations. Priority was given to favorably placed GRB fields, so high $(>2)$ airmass targets were in some cases skipped. A discussion of the completeness of our sample can be found in Section 5. Images were taken in the Bessel $B$-, $V$-, and $R$-band, as well as Gunn $i$-band. We mostly took $600 \mathrm{~s}$ exposures which later were combined and stacked; the first four columns of Table 2 summarizes the data set and quote total exposure time and average seeing values in the different filters for the 24 GRB fields in this sample. Unless otherwise noted, all observations were performed well after the optical afterglow had faded.

\section{Reductions}

\subsection{Pre-processing and stacking}

The images were pre-processed in the standard way using IRAF (Image Reduction and Analysis Facility). The bias level was subtracted using the overscan region on each science frame and a master bias frame computed from typically 17-20 individual frames on each night. Flat-fielding was performed exclusively by means of sky flats. Dark current did not need to be corrected for as the dark level was found to be totally negligible. Images taken in the $i$-band (and to some extent the $R$-band) were affected by fringing. We tried to minimize the effect on the photometry by carefully selecting the region from which the local sky level was estimated. The $i$-band images also suffered from a large gradient in the background level across the CCD frame (from upper left to lower right corner), 
again making careful local background estimation important.

Individual frames were stacked in IRAF with offsets according to the World Coordinate System (WCS) keywords in the image headers. However, the original WCS information was incorrect and had to be re-computed first. We used SExtractor Bertin \& Arnouts 1996) for source detection on the individual images and then correlated the pixel positions with sky coordinates from the USNOB1.0 Catalog (Monet et al. 2003) using the imwcs program in WCSTools 2 The WCS fit was typically derived from 100-200 objects in the generous $13.7^{\prime}$ field of view and allowed for a robust astrometric calibration. Images with high sky values and/or short exposure times were down-weighted during the stacking. Specifically, in IRAF's IMCOMBINE task the frames were scaled and weighted by the exposure time, and the zero level offset was taken as the mode within a central region of the CCD frame.

\subsection{Photometry}

For photometry we used an IDL (Interactive Data Language) code (see e.g. Ovaldsen et al. 2003a, b) featuring circular or elliptical apertures with correction for fractional pixels at the aperture border. Centering was performed using paraboloidal representation of the nine central pixel values of the source in question (Teuber 1993). The local background level was calculated from several apertures arranged around the object of interest, and any apertures containing cosmics, sources etc. were automatically discarded.

The photometric calibration was performed relative to the Landolt $\left(B V R_{C} I_{C}\right)$ system. Zero-points, extinction coefficients and color terms were derived from observations of Lan-

\footnotetext{
${ }^{1}$ The accuracy of the USNO-B1.0 Catalog is 0.2 . ${ }^{2}$ http://tdc-www.cfa.harvard.edu/software/wcstools/
}

dolt standard fields on two nights with photometric conditions. Color terms were negligible for all filters, except $B$ (the color-correction term, $c_{B}(B-V)$, had $\left.c_{B}=-0.12\right)$. Each GRB field was calibrated using three to five comparison stars.

We quote a detection limit, or upper limit, for all GRBs in the sample. This parameter is a function of (at least) background noise and seeing. $2 \sigma$-limits were calculated in the following way:

$$
\operatorname{Mag}(2 \sigma)=\mathrm{ZP}-2.5 \log \left(2 C \sqrt{\pi r_{d}^{2} \sigma_{B}^{2}}\right),
$$

where $\mathrm{ZP}$ is the photometric zero-point, $C$ is the aperture correction, $r_{d}$ is the radius of the circular detection aperture (see below), and $\sigma_{B}$ is a conservative measure of the background noise, i.e. the standard deviation of the sky values. $C$ is the ratio between the total flux of the reference stars and the flux inside the detection aperture whose radius, $r_{d}$, is set to 1.5 times the half width at half maximum (HWHM). The HWHM was measured from the point-spread function (PSF) on each image. The factor two inside the parenthesis corresponds to a $2 \sigma$-limit.

\section{Results}

The stacked images of the 24 GRB fields were examined with respect to objects in or near the Swift XRT error circle and other reported positions, i.e. from afterglow observations in various bands mainly from ground-based telescopes. We use the new, refined Swift XRT positions and errors presented in Butler (2006), which are determined by matching X-ray field source positions directly to those of counterpart optical sources. We also note that several SwiftUVOT (UV/Optical Telescope) positions for the afterglows are inconsistent with the refined XRT error circle and OT positions from other groups. A detection limit, i.e. upper 
limit for the brightness of the host, was derived for each GRB field, and photometry was performed for the cases where we found probable host galaxy candidates.

Each object in our GRB sample is treated below; please consult Fig. 1. Table 1 and Table 2 for details about the target GRBs, the data (filter, exposure time, and seeing) and for $2 \sigma$ detection limits, photometry and positions of host galaxy candidates. The upper limits and host galaxy magnitudes in Table 2 are not corrected for foreground Galactic extinction. The reader will also find that the detection limit (column 5) typically is different for images with roughly the same exposure time and seeing; this is mainly due to differences in moon illumination and sky brightness. Quite often positions of afterglow candidates in $\mathrm{GCN}^{3}$ circulars are lacking error estimates, and in such cases we adopt a 0.5 error radius in the figures. Also, we only include optical afterglow positions which have been reported to be transient. The tables, the mosaic figure (from upper left to lower right) and the following subsections are all arranged chronologically. Throughout this paper we use a $\left(\Omega_{\mathrm{m}}, \Omega_{\Lambda}\right)=(0.3,0.7)$ cosmology with Hubble Constant $H_{0}=70 \mathrm{~km} \mathrm{~s}^{-1} \mathrm{Mpc}^{-1}$.

\footnotetext{
${ }^{3}$ Gamma-ray burst Coordinates Network
} 
TABLE 1

GRB TARGET LIST

\begin{tabular}{|c|c|c|c|c|c|c|c|c|}
\hline \multirow[t]{2}{*}{ GRB } & \multirow[t]{2}{*}{ Redshift } & \multicolumn{3}{|c|}{ XRT position $(\mathrm{J} 2000)^{\mathrm{a}}$} & \multicolumn{4}{|c|}{ OT position (J2000) } \\
\hline & & $\mathrm{RA}$ & Dec & Error & RA & Dec & Error & Ref. \\
\hline \multirow[t]{2}{*}{050318} & $1.44[1]$ & 03:18:51.04 & $-46: 23: 43.5$ & 2.7 & 03:18:51.0 & $-46: 23: 44$ & 0.5 & [11] \\
\hline & & & & & 03:18:51.15 & $-46: 23: 43.7$ & 0.3 & {$[12]$} \\
\hline \multirow[t]{2}{*}{050401} & $2.90[2]$ & $16: 31: 28.84$ & $+02: 11: 14.5$ & 1.8 & $16: 31: 28.82$ & $+02: 11: 14.83$ & 0.5 & [13] \\
\hline & & & & & $16: 31: 28.81$ & $+02: 11: 14.2$ & $\cdots$ & {$[14]$} \\
\hline \multirow[t]{2}{*}{050406} & $\ldots$ & $02: 17: 52.25$ & $-50: 11: 15.0$ & 1.3 & $02: 17: 52.3$ & $-50: 11: 15$ & 0.5 & [15] \\
\hline & & & & & $02: 17: 52.2$ & $-50: 11: 15.8$ & $\ldots$ & {$[16]$} \\
\hline 050412 & $\ldots$ & $12: 04: 25.18$ & $-01: 12: 00.8$ & 6.9 & $\cdots$ & $\ldots$ & $\cdots$ & \\
\hline \multirow[t]{2}{*}{050416} & $0.65[3]$ & $12: 33: 54.57$ & $+21: 03: 26.9$ & 0.6 & $12: 33: 54.6$ & $+21: 03: 26.7$ & $\cdots$ & [17] \\
\hline & & & & & $12: 33: 54.56$ & $+21: 03: 27.73$ & $\ldots$ & [18] \\
\hline 050502B & $\ldots$ & 09:30:10.06 & $+16: 59: 46.5$ & 1.0 & 09:30:10.024 & $+16: 59: 48.07$ & $\cdots$ & [19] \\
\hline \multirow[t]{2}{*}{050603} & $2.82[4]$ & 02:39:56.90 & $-25: 10: 55.7$ & 0.9 & 02:39:56.891 & $-25: 10: 54.6$ & 0.1 & [20] \\
\hline & & & & & 02:39:56.839 & $-25: 10: 54.92$ & $\ldots$ & [21] \\
\hline 050607 & $\cdots$ & $20: 00: 42.77$ & $+09: 08: 31.1$ & 1.4 & 20:00:42.79 & $+09: 08: 31.5$ & 0.5 & [22] \\
\hline 050714B & $\ldots$ & $11: 18: 47.75$ & $-15: 32: 49.3$ & 2.1 & $\ldots$ & $\ldots$ & $\ldots$ & \\
\hline 050726 & $\cdots$ & $13: 20: 12.16$ & $-32: 03: 51.0$ & 3.7 & $13: 20: 11.9$ & $-32: 03: 51.9$ & $\cdots$ & [23] \\
\hline \multirow[t]{2}{*}{050801} & $\ldots$ & $13: 36: 35.51$ & $-21: 55: 42.7$ & 5.0 & $13: 36: 35.4$ & $-21: 55: 42.0$ & $\ldots$ & {$[24]$} \\
\hline & & & & & $13: 36: 35.363$ & $-21: 55: 42.03$ & $\cdots$ & [25] \\
\hline 050822 & $\cdots$ & $03: 24: 27.22$ & $-46: 02: 00.0$ & 0.7 & $\ldots$ & $\ldots$ & $\cdots$ & \\
\hline 050826 & $0.30[5]$ & 05:51:01.69 & $-02: 38: 37.6$ & 2.6 & 05:51:01.58 & $-02: 38: 35.8$ & 0.5 & {$[26]$} \\
\hline 050908 & $3.34[6]$ & 01:21:50.85 & $-12: 57: 17.9$ & 2.2 & $01: 21: 50.75$ & $-12: 57: 17.2$ & 0.3 & {$[27]$} \\
\hline 050915 & $\ldots$ & $05: 26: 44.86$ & $-28: 00: 59.9$ & 1.4 & $05: 26: 44.804$ & $-28: 00: 59.27$ & 0.18 & [28] \\
\hline 050922C & $2.20[7]$ & 21:09:33.12 & $-08: 45: 28.3$ & 2.0 & 21:09:33.083 & $-08: 45: 30.2$ & 0.2 & [29] \\
\hline 051006 & $\ldots$ & $07: 23: 14.03$ & $+09: 30: 21.9$ & 4.3 & $\ldots$ & $\ldots$ & $\ldots$ & \\
\hline 051016 & $\ldots$ & 08:11:16.77 & $-18: 17: 53.7$ & 2.2 & $\ldots$ & $\ldots$ & $\ldots$ & \\
\hline 051016B & $0.94[8]$ & 08:48:27.80 & $+13: 39: 20.7$ & 0.9 & $08: 48: 27.81$ & $+13: 39: 20.0$ & $\ldots$ & {$[30]$} \\
\hline \multirow[t]{2}{*}{$051021^{\dagger}$} & $\ldots$ & $01: 56: 36.5$ & $+09: 04: 06.1$ & 4.0 & $01: 56: 36.37$ & $+09: 04: 03.27$ & 0.5 & {$[31]$} \\
\hline & & & & & 01:56:36.39 & $+09: 04: 03.7$ & 0.5 & {$[32]$} \\
\hline $051022^{\dagger}$ & $0.8 \quad[9]$ & 23:56:04.1 & $+19: 36: 25.1$ & 4.0 & 23:56:04.1 & $+19: 36: 24.1$ & 1.0 & {$[33]^{\ddagger}$} \\
\hline 051117B & $\ldots$ & $05: 40: 43.21$ & $-19: 16: 27.2$ & 2.0 & $\ldots$ & $\ldots$ & $\ldots$ & \\
\hline 060223 & $4.41[10]$ & 03:40:49.82 & $-17: 07: 49.8$ & 3.4 & 03:40:49.55 & $-17: 07: 48.36$ & 1.0 & [34] \\
\hline 060313 & & 04:26:28.41 & $-10: 50: 40.7$ & 2.4 & $04: 26: 28.4$ & $-10: 50: 40.1$ & 0.5 & {$[35]$} \\
\hline
\end{tabular}

NotE.-XRT and OT positions (with error radii in arcsec) for the GRBs in our sample, see also Fig. 1] References: [1] Berger \& Mulchaev (2005), [2] Fvnbo et al. (2005a), [3] Cenko et al. (2005c), [4] Berger \& Becker (2005), [5] Halpern et al. (2006b), [6] Prochaska et al. (2005), [7] Jakobsson et al. (2005b), [8] Soderberg et al. (2005b), [9] Gal-Yam et al. (2005), [10] Berger et al. (2006), [11] Mulchaey \& Berger (2005), [12] de Pasquale et al. (2005), [13] Soderberg et al. (2005a), [14] McNaught et al. (2005), [15] Berger et al. (2005), [16] Rol et al. (2005), [17] Cenko et al. (2005a), [18] Schadv et al. (2005), [19] Rich et al. (2005), [20] Cameron et al. (2005), [21] Brown et al. (2005), [22] Rhoads et al. (2005), [23] Poole et al. (2005), [24] Rykoff et al. (2005), [25] Fynbo et al. (2005b), [26] Halpern et al. (2006a), [27] Li et al. (2005), [28] Bloom (2005), [29] Jakobsson et al. (2005a), [30] Chen et al. (2005a), [31] Rumvantsev et al. (2005), [32] Fox et al. (2005b), [33] Cameron \& Frail (2005), [34] Cummings et al. (2006), [35] Levan et al. (2006).

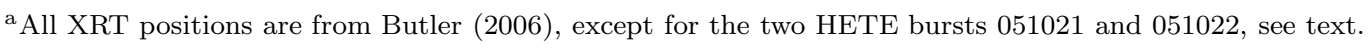

${ }^{\dagger} H E T E$-localized burst (the rest are Swift bursts)

$\ddagger$ Radio transient 
TABLE 2

DATA SET AND PHOTOMETRY

\begin{tabular}{|c|c|c|c|c|c|c|c|}
\hline GRB & Band $^{a}$ & Exp. time ${ }^{b}$ & Seeing $^{\mathrm{c}}$ & $2 \sigma$-limit ${ }^{\mathrm{d}}$ & Host candidate & $\mathrm{RA} \& \operatorname{Dec}(\mathrm{J} 2000)^{\mathrm{e}}$ & $\mathrm{OT}^{\mathrm{f}}$ \\
\hline 050318 & $R$ & 36000 & 1.0 & 26.0 & $\ldots$ & $\ldots$ & $\mathrm{y}$ \\
\hline \multirow[t]{2}{*}{050401} & $R$ & 7200 & 1.2 & 25.1 & $\cdots$ & 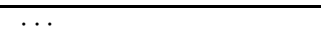 & $\mathrm{y}$ \\
\hline & $V$ & 14000 & 1.15 & 25.7 & $\ldots$ & $\ldots$ & \\
\hline 050406 & $R$ & 8100 & 1.2 & 25.1 & $\cdots$ & $\cdots$ & $\mathrm{y}$ \\
\hline \multirow[t]{3}{*}{050412} & $R$ & 5400 & 1.75 & 24.6 & $22.4(0.2)$ & $12: 04: 25.03-01: 12: 04.0$ & $\mathrm{n}$ \\
\hline & $I$ & 3000 & 0.8 & 24.3 & $21.6(0.1)$ & $\ldots$ & \\
\hline & $V$ & 8250 & 1.05 & 25.2 & $23.4(0.2)$ & $\ldots$ & \\
\hline 050416 & $V$ & 15900 & 1.3 & 25.8 & $24.2(0.2)$ & $12: 33: 54.59+21: 03: 26.6$ & $\mathrm{y}$ \\
\hline $050502 \mathrm{~B}$ & $\bar{V}$ & 31400 & 1.1 & 26.3 & $\cdots$ & $\cdots$ & $\mathrm{y}$ \\
\hline \multirow[t]{2}{*}{050603} & $R$ & 17400 & 1.15 & 25.4 & $\cdots$ & $\cdots$ & $\mathrm{y}$ \\
\hline & $I$ & 7200 & 1.0 & 24.4 & $\cdots$ & $\cdots$ & \\
\hline \multirow[t]{2}{*}{050607} & $R$ & 1980 & 1.1 & 24.0 & $\cdots$ & 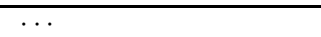 & $\mathrm{y}$ \\
\hline & $I$ & 2400 & 1.55 & 22.6 & $\ldots$ & $\ldots$ & \\
\hline \multirow[t]{4}{*}{ 050714B } & $R$ & 15000 & 1.15 & 25.8 & $21.8(0.1)$ & $11: 18: 47.72-15: 32: 51.6$ & $\mathrm{n}$ \\
\hline & $I$ & 8700 & 0.95 & 24.3 & $20.6(0.1)$ & $\cdots$ & \\
\hline & $V$ & 27400 & 1.05 & 26.3 & $22.9(0.1)$ & $\ldots$ & \\
\hline & $B$ & 15600 & 1.1 & 26.1 & $24.9(0.3)$ & $\ldots$ & \\
\hline 050726 & $\bar{V}$ & 15900 & 1.0 & 25.9 & $\cdots$ & $\cdots$ & $\mathrm{y}$ \\
\hline \multirow[t]{3}{*}{050801} & $I$ & 65550 & 1.15 & 24.8 & $\cdots$ & 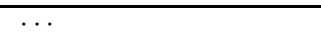 & $\mathrm{y}$ \\
\hline & $V$ & 22500 & 1.1 & 26.2 & $\ldots$ & $\cdots$ & \\
\hline & $B$ & 24000 & 1.05 & 26.3 & $\ldots$ & $\ldots$ & \\
\hline 050822 & $\bar{V}$ & 9600 & 1.7 & 25.0 & $\cdots$ & 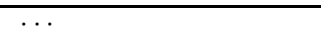 & $\mathrm{n}$ \\
\hline \multirow[t]{4}{*}{050826} & $R$ & 27000 & 0.95 & 25.8 & $21.4(0.1)$ & $05: 51: 01.59-02: 38: 36.1$ & $\mathrm{y}$ \\
\hline & $I$ & 3000 & 1.0 & 23.8 & $20.6(0.2)$ & .. & \\
\hline & $V$ & 6000 & 1.1 & 25.6 & $22.4(0.2)$ & $\cdots$ & \\
\hline & $B$ & 5400 & 1.1 & 25.4 & $23.9(0.3)$ & $\ldots$ & \\
\hline 050908 & $R$ & 3000 & 0.75 & 25.2 & $\cdots$ & $\cdots$ & $\mathrm{y}$ \\
\hline \multirow[t]{4}{*}{050915} & $R$ & 22000 & 0.95 & 25.9 & $24.8(0.4)$ & $05: 26: 44.84-28: 00: 59.7$ & $\mathrm{y}$ \\
\hline & $I$ & 10800 & 1.1 & 24.3 & $\ldots$ & . . & \\
\hline & $V$ & 13800 & 1.0 & 25.9 & $25.2(0.5)$ & $\cdots$ & \\
\hline & $B$ & 3000 & 1.1 & 25.5 & $\ldots$ & $\ldots$ & \\
\hline $050922 \mathrm{C}$ & $R$ & 23700 & 0.95 & 25.8 & $\cdots$ & $\cdots$ & $\mathrm{y}$ \\
\hline \multirow[t]{4}{*}{051006} & $R$ & 9600 & 0.95 & 25.6 & $23.0(0.1)$ & $07: 23: 14.10+09: 30: 20.2$ & $\mathrm{n}$ \\
\hline & $I$ & 5400 & 0.9 & 24.3 & $22.2(0.2)$ & $\cdots$ & \\
\hline & $V$ & 7200 & 1.1 & 25.6 & $23.3(0.2)$ & $\cdots$ & \\
\hline & $B$ & 6600 & 1.1 & 25.6 & $24.2(0.2)$ & $\cdots$ & \\
\hline 051016 & $R$ & 24900 & 0.95 & 25.6 & $\cdots$ & l. & $\mathrm{n}$ \\
\hline $051016 \mathrm{~B}$ & $V$ & 16800 & 1.05 & 26.1 & $23.1(0.2)$ & $08: 48: 27.84+13: 39: 20.2$ & $\mathrm{y}$ \\
\hline \multirow[t]{4}{*}{$051021^{\dagger}$} & $R$ & 19800 & 1.1 & 25.4 & $24.9(0.4)$ & $01: 56: 36.35+09: 04: 03.7$ & $\mathrm{y}$ \\
\hline & $I$ & 11400 & 1.2 & 24.0 & $\ldots$ & . & \\
\hline & $V$ & 6000 & 1.3 & 25.2 & $\cdots$ & $\cdots$ & \\
\hline & $B$ & 1800 & 1.25 & 24.6 & $\ldots$ & . & \\
\hline \multirow[t]{4}{*}{$051022^{\dagger}$} & $R$ & 15000 & 1.0 & 25.4 & $21.7(0.1)$ & $23: 56: 04.10+19: 36: 24.2$ & $\mathrm{n}$ \\
\hline & $I$ & 13800 & 1.3 & 23.8 & $21.1(0.1)$ & $\cdots$ & \\
\hline & $V$ & 12600 & 1.15 & 25.7 & $22.3(0.1)$ & $\ldots$ & \\
\hline & $B$ & 12600 & 1.2 & 25.7 & $22.8(0.2)$ & $\ldots$ & \\
\hline \multirow[t]{3}{*}{ 051117B } & $R$ & 10200 & 1.1 & 25.1 & $21.0(0.1)$ & $05: 40: 43.29-19: 16: 26.1$ & $\mathrm{n}$ \\
\hline & $I$ & 13650 & 1.15 & 24.0 & $20.4(0.1)$ & $\ldots$ & \\
\hline & $V$ & 9000 & 1.0 & 25.2 & $21.8(0.2)$ & $\ldots$ & \\
\hline
\end{tabular}


TABLE 2-Continued

\begin{tabular}{lrrlllll}
\hline \hline GRB & Band $^{\mathrm{a}}$ & Exp. time $^{\mathrm{b}}$ & Seeing $^{\mathrm{c}}$ & $2 \sigma$-limit & Host candidate & RA \& Dec $(\mathrm{J} 2000)^{\mathrm{e}}$ & OT $^{\mathrm{f}}$ \\
\hline & $B$ & 12600 & 1.0 & 25.3 & $22.5(0.2)$ & $\cdots$ & \\
\hline 060223 & $R$ & 14600 & 1.2 & 25.3 & $\cdots$ & $\cdots$ & $\mathrm{y}$ \\
\hline 060313 & $R$ & 8100 & 1.30 & 23.9 & $\cdots$ & $\cdots$ & $\mathrm{y}$ \\
\hline
\end{tabular}

NotE.-Details regarding the data set and our photometric results. The host galaxy candidates for GRBs 050915 and 051021 are new and have not been suggested or published by others.

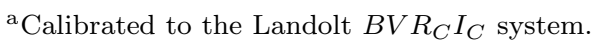

${ }^{\mathrm{b}}$ Total exposure time (seconds) of stacked image.

${ }^{\mathrm{c}}$ FWHM seeing (arcseconds) of stacked image.

${ }^{\mathrm{d}}$ See definition of detection limit in Sect. 3.2

eEstimated errors are 0. . 5 in both coordinates.

${ }^{\mathrm{f}}$ Optical transient (yes or no), see also Table 1

${ }^{\dagger}$ HETE-localized burst 


\subsection{GRB 050318}

Sixty $600 \mathrm{~s}$ exposures, some under bright sky conditions, yield a detection limit of $R=26.0$ for this $z=1.44$ burst (Krimm et al. 2005; Berger \& Mulchaev 2005). The optical afterglow positions reported by de Pasquale et al. 2005, Swift-UVOT) and Mulchaey \& Berger (2005) are not fully consistent, however both are inside the refined XRT error circle of Butler (2006).

We do not find any sources in immediate proximity to the Swift-UVOT error circle of de Pasquale et al., but an extended source is seen at the border of the XRT error circle, less than $2^{\prime \prime}$ west of the Mulchaey \& Berger position (see Fig. 1). The light distribution has two peaks a little more than $1^{\prime \prime}$ apart, so it could potentially be two separate (possibly interacting) galaxies or two bright spots (e.g. star-forming regions) in the same galaxy. Using an elliptical aperture around the entire light distribution, we estimate the magnitude to be $R=23.8 \pm 0.2$. However, we err on the side of conservatism and exclude this source as a host candidate, since OTs typically are highly concentrated on the very brightest regions of their host galaxies (e.g. Fruchter et al. 2006). At $z=1.44$ the separation between the source (maximum pixel) and the OT is about $16 \mathrm{kpc}$, which is considered too large in terms of long bursts whose progenitors are assumed to be massive stars. If the error bar on the reported OT position of Mulchaey \& Berger (2005) was larger, the object found here could qualify as a host candidate.

\subsection{GRB 050401}

GRB 050401 (Angelini et al. 2005; de Pasquale et aW. as not the OT. We regard this extended ob2006) is a high-redshift burst; Fvnbo et al. (2005a) obtained spectra of the afterglow and found $z=2.90$ from several absorption lines (see also Watson et al. 2006). Optical and radio afterglow positions are reported by McNaught et al. (2005) and Soderberg et al. (2005a), respectively. Our limits of $R=25.1$ and $V=25.7$ are not deep enough to reveal any host galaxy.

\subsection{GRB 050406}

Two afterglow positions (Berger et al. 2005; Rol et al. 2005) were reported for GRB 050406 (Parsons et al. 2005a), and both are shown in Fig. 1. No sources are found in or near the XRT error circle down to the limiting magnitude of $R=25.1$.

\subsection{GRB 050412}

The field around GRB 050412 (Cummings et al. 2005) is contaminated by a bright $R=11.3$ star (USNO U0825-07638162) approximately $50^{\prime \prime}$ north-east. No transient source has been confirmed in optical bands, although Jensen et al. (2005) and Fox et al. (2005a) detected a single source and quoted approximate values of $R \sim 22$ (1.5 h after the burst) and $R \gtrsim 21.5$ (55 min after the burst), respectively. Later, Kosugi et al. (2005) found another source inside the XRT error circle estimated at $R=26.0 \pm 0.5$, but was unable to determine whether it was point-like or extended.

We find a clearly extended object inside the XRT error circle. VRI-photometry and position are listed in Table 2. In particular, our measurement of $R=22.4 \pm 0.2$ are in rough agreement with Jensen et al. (2005) and Fox et al. (2005a), considering their very preliminary and approximate values, and the positions are also coincident. This suggests that the source reported by the above authors ject as a possible host galaxy.

Unfortunately, our images are not deep enough to probe the faint $R=26.0 \pm 0.5$ detection by Kosugi et al. (2005). Further imag- 


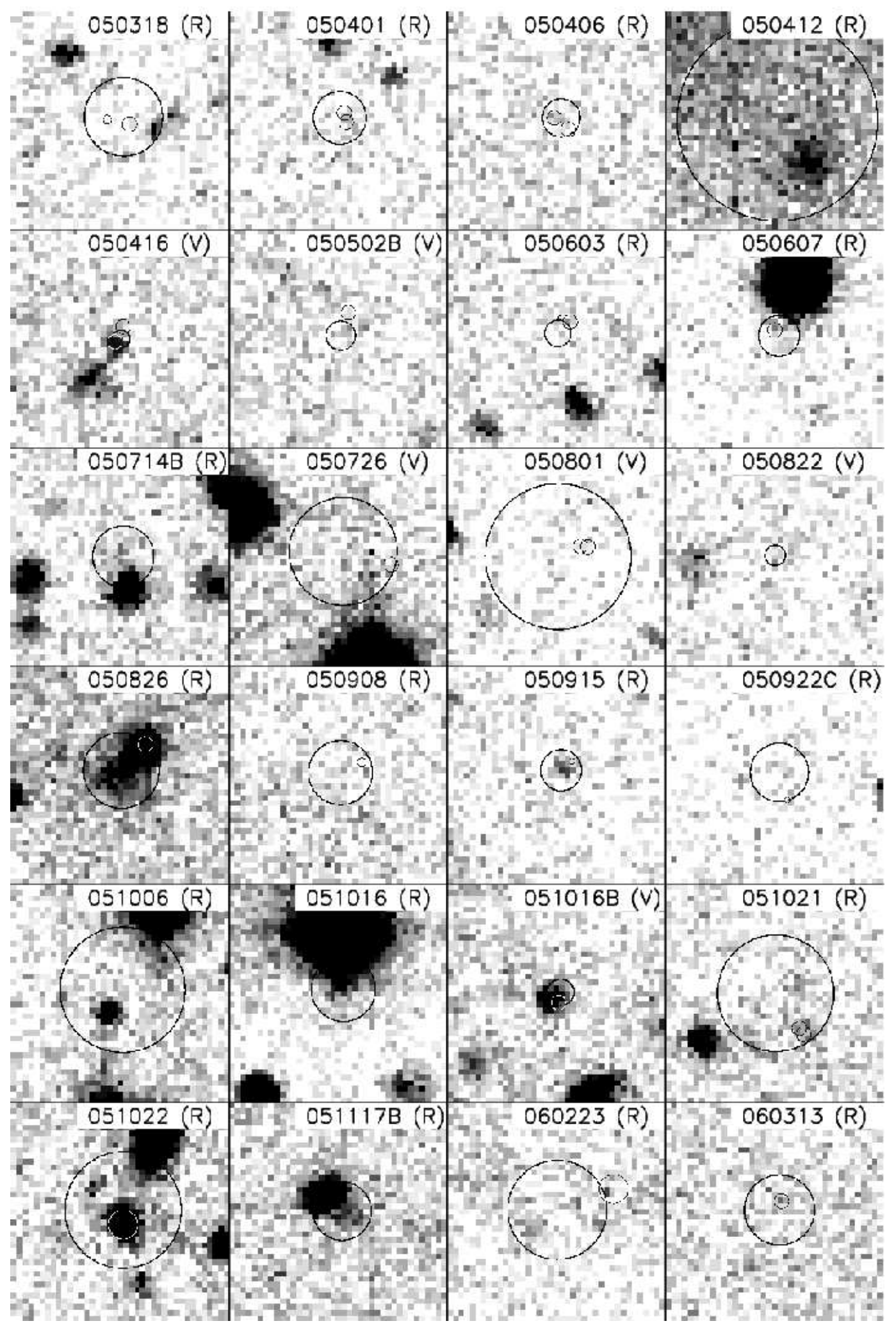

Fig. 1.- Our GRB sample. Thick, large circles are the (refined) Swift XRT error circle and any smaller circles correspond to reported (optical, IR or radio) afterglow positions, see the text and Table 1. Each image is $15^{\prime \prime} \times 15^{\prime \prime}$, with greyscale cuts of ${ }_{-0.5}^{+5} \sigma_{B}$. The seeing, exposure time and $2 \sigma$-detection limit for each GRB field are found in Table 2. Several OT positions are lacking error estimates, see Table 1, in these cases we plot a 0.5 error radius. For the figures, the original frames were rotated and resampled in order to make North up and East to the left. 
ing to search for this object, as well as spectroscopy of the host candidate presented here, are advised.

\subsection{GRB 050416}

An optical afterglow for GRB 050416 (Sakamoto et al. 2005) was first found by Cenko et al. (2005a) and later confirmed by Schady et al. (2005) and other groups, also at IR and radio wavelengths. Cenko et al. (2005c) used Keck I spectra to calculate a redshift of $z=0.6535$ from the host galaxy's emission lines. See also Soderberg et al. (2006) and Holland et al. (2007).

Our $V$-band image clearly detects the galaxy (along with another extended source outside the XRT error circle), see Fig. 1, The magnitude is estimated to be $V=24.2 \pm 0.2$ and the position is given in Table 2 ,

\subsection{GRB 050502B}

We only carried out $V$-band imaging for GRB 050502B (Falcone et al. 2005, 2006) and reached a limit of 26.3 mag. The stacked image shows no sign of any host near the Rich et al. (2005) candidate afterglow position (transient was only seen in the $I$-band) or inside the XRT circle.

\subsection{GRB 050603}

GRB 050603 (Retter et al. 2005a; Grupe et al. 2006) have both optical (Berger \& McWilliam 2005; Brown et al. 2005) and radio (Cameron et al. 2005) afterglows, but no imaging of the host galaxy. 2.13 days after the burst Berger \& Becker (2005) found a redshift of $z=2.821$ based on a single bright emission line interpreted as Ly $\alpha$.

Our $R$ - and $I$-band images, with limits 25.4 mag and $24.4 \mathrm{mag}$, respectively, do not show any sources near the afterglow positions.

\subsection{GRB 050607}

The host of GRB 050607 (Retter et al. 2005b; Pagani et al. 2006b) will be hard to study due to the nearby $R=16.6$ USNO star U0975-17511046, see Fig. 1, Our rather shallow limits of $R=24.0$ and $I=22.6$ do not reveal any other objects near the afterglow position of Rhoads et al. (2005). PSFsubtraction is difficult as the star is saturated in our images.

\subsection{GRB 050714B}

GRB 050714B (Levan et al. 2005) had an initial XRT error circle where four sources reportedly where candidates for either the OT or host galaxy. The refined XRT position of Butler (2006) only includes one of them, i.e. source \#1 from Covino et al. (2005, see also Malesani et al. 2005), which was found to be non-variable with an estimated $R=21.7 \mathrm{mag}$. None of the reported sources have been confirmed to fade and are thus not considered as OT candidates. Additionally, we detect all of them in our images taken months after the burst.

Down to the limits given in Table2, we only detect the object mentioned above (partly) inside the refined XRT error circle. We consider it a host candidate, although $B V R I$ photometry yields a very red color and it is unresolved at the resolution of the images. Our $R$-band magnitude of $21.8 \pm 0.1$ agrees with Covino et al. (2005).

\subsection{GRB 050726}

Poole et al. (2005) reported a detection for this burst (Barthelmy et al. 2005) by SwiftUVOT only in the $V$-band, with $V=17.35 \pm$ 0.09 at $123 \mathrm{~s}$ after the burst, and then dropping below the detection threshold. This detection has not been confirmed by others.

South-east of the Swift-UVOT position our $V$-band image (taken months after the burst) 
shows a few pixels with values above the background level, see Fig. 1. However, at least some of this signal is due to a diffraction spike from the nearby star. Thus is not possible to say whether this is a very weak detection of the host galaxy. The limiting magnitude for this image is $V=25.9$.

\subsection{GRB 050801}

The ROTSE collaboration reported the first optical afterglow (Rykoff et al. 2005, 2006) of Swift trigger 148522. Later, Fynbo et al. $(2005 \mathrm{~b})$ found an OT position consistent with Rykoff et al. (2005), while the position from Swift-UVOT (Blustin et al. 2005a) is at variance with the aforementioned positions and even outside the XRT error circle. We do not detect any host candidates in the immediate surroundings, despite rather deep limits as far as our observations are concerned, see Table 2 and Fig. 1,

\subsection{GRB 050822}

Our images of GRB 050822 (Blustin et al. 2005b; Godet et al. 2007) were taken in rather poor seeing and yielded an upper limit of $V=25.0$. With the exception of the X-ray band, there have been no reports concerning transient objects.

\subsection{GRB 050826}

Halpern et al. (2005) claim the detection of a fading optical afterglow for GRB 050826 (Mangano et al. 2005) located at the border of the refined XRT position, see Fig. 1, From August 26 to 27 Halpern et al. measure a decrease in brightness from $R=21.0$ to $R=21.6$ (both $\pm 0.2 \mathrm{mag}$ ). In a later $\mathrm{GCN}$, Halpern et al. (2006a) provide positions for the presumed host galaxy and OT candidate, and recently the redshift of the host candidate was measured to be $z=0.297$ (Halpern et al. 2006b).
In our 27000 s stacked $R$-image we find an extended source with magnitude $R=21.4 \pm$ 0.1. The source seems to consist of a conspicuously point-like object (position: see Table 2) plus an extended, irregular and much fainter source in terms of maximum pixel intensity. The resolved part lies in the very center of the refined XRT error circle. The quoted $B V R I$-magnitudes in the table were found using elliptical apertures covering the entire light distribution. We note that our position for the point-like source is consistent with both the OT and host position of Halpern et al. (2006a).

This field is the only one in our sample with a significant foreground Galactic extinction: $E_{B-V}=0.6$ according to the dust maps of Schlegel et al. (1998).

\subsection{GRB 050908}

The observations of GRB 050908 (Goad et al. 2005) comprise only five $600 \mathrm{~s}$ exposures, but favorable seeing yields a detection limit of $R=25.2$. OT positions are provided by Torii (2005) and Li et al. (2005); the latter one is plotted in Fig. 1. Given a burst redshift of $z=3.344$ (Prochaska et al. 2005), it is not surprising that the host galaxy is undetected in our image.

\subsection{GRB 050915}

An IR counterpart ( $H$-band, Bloom 2005) has been observed for GRB 050915 (Grupe et al. 2005), but no redshift or host galaxy has been found. However, close to the IR afterglow position by Bloom both our $R$ - and $V$-band images reveal 7 pixels above the $2 \sigma$ noise level, see Fig. 1. Images in filters $I$ and $B$ have limiting magnitudes of 24.3 and 25.5, respectively, and yield no signal.

Aperture photometry is highly uncertain for such a marginal detection. However, if we assume that the light distribution of the 
host candidate follows the PSF, one can construct a synthetic model and resample it on the pixel array nearby the candidate. The strength of the synthetic source is increased until the detected light is similar to the host candidate. This gives $R=24.8 \pm 0.4$ and $V=25.2 \pm 0.5$. The errors are obviously rather large and this method rests on the assumption that the brightness profile of the detected part of the host is similar to the image PSF. However, a distant and/or faint galaxy might easily fulfill this assumption.

\subsection{GRB 050922C}

For GRB 050922C (Norris et al. 2005a) we have a total of $23700 \mathrm{~s}$ in the $R$-band, giving a detection limit of $R=25.8$. The host at $z=2.198$ (Jakobsson et al. 2005b, redshift based on absorption features in the optical afterglow) must be fainter than this limit as our observations fail to detect anything inside the XRT error circle and, more specifically, at the well-constrained optical afterglow position of Jakobsson et al. (2005a).

\subsection{GRB 051006}

Inside the XRT error circle of GRB 051006 (Norris et al. 2005b) we find in our images a point-like source. This is the same object as the "\#2 source" mentioned by de Ugarte et al. (2005), who was not able to check for variability and did not estimate the brightness. (Regarding the other objects in early reports: source \#1 is a USNO star and source \#3 is about 5 " outside the refined XRT error circle.) In the absence of a confirmed optical/IR/radio afterglow, it seems that the object mentioned by de Ugarte et al. (2005) was an existing source, for which we here present multi-color photometry (Table 22). We consider it a host candidate. It is the only detected object inside the XRT error circle.

\subsection{GRB 051016}

We do not detect any sources inside the XRT error circle of GRB 051016 (Boyd et al. 2005) down to a limiting magnitude of $R=$ 25.6. No OTs are reported. As seen in the figure, a $R=16.7$ star (USNO U0675-08481584) contaminates the area, and PSF-subtraction of this partly saturated star reveals no apparent host candidates.

\subsection{GRB 051016B}

Soderberg et al. (2005b) found a redshift of $z=0.9364$ for GRB 051016B (Parsons et al. 2005b) after observing the OT position by Chen et al. (2005a) about 15 days after the burst. The redshift determination was based on two emission lines ([O II $]$ and Ne III) in spectra from the Keck I telescope.

We here present the first image of the host galaxy, which is unresolved in our images, see Fig. 1. It is located near the OT position of Chen et al., and we measure $V=23.1 \pm 0.2$ mag.

\subsection{GRB 051021}

This HETE burst (Yoshida et al. 2005) has no reported redshift or host galaxy. At the position of the optical afterglow (Fox et al. 2005b; Rumvantsev et al. 2005) we detect $4-5$ neighboring pixels with values at the $2 \sigma$ noise level in our stacked $R$-band image, see Fig. 1 . Formally, this detection is of low significance, but combining all data from all four bands strengthens the presence of an excess flux on this area. Moreover, Gaussian smoothing of the $R$-band image reveals a faint, but significant source just at the OT position, see Fig. 2, We estimate $R=24.9 \pm 0.4$ for this likely host galaxy of GRB 051021 .

\subsection{GRB 051022}

Despite extensive observational efforts from many groups no optical afterglow was 


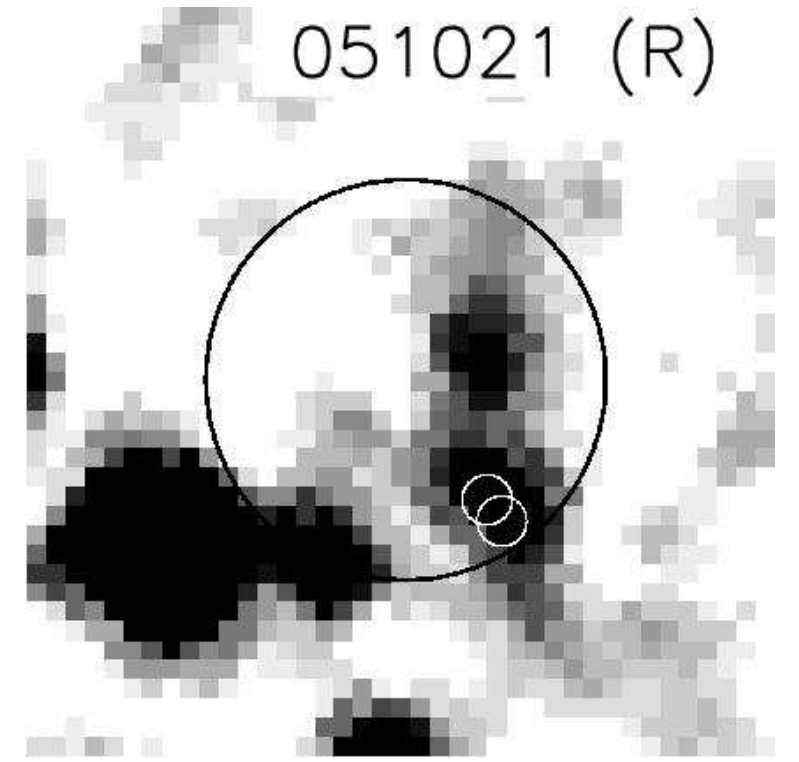

Fig. 2.- Smoothed $R$-band image of GRB 051021. The two small circles correspond to the OT positions mentioned in the text and in Table 1 .

found for this HETE burst (Olive et al. 2005; Nakagawa et al. 2006). However, the host galaxy was pinpointed and a redshift of $z=0.8$ was deduced from preliminary reductions where a strong line was interpreted as OII (Gal-Yam et al. 2005). Cameron \& Frail (2005) also found a candidate radio afterglow coincident with the host galaxy (see Fig. 1). In Table 2 we present $(B V R I)$ photometry for the host galaxy of GRB 051022 .

\subsection{GRB 051117B}

GRB 051117B (Band et al. 2005) has no known optical counterpart or redshift. A possible host was suggested by Thöne et al. (2005, see also Chen et al. 2005b) using part of the data presented in this article.

In Table 2 we present BVRI photometry and a refined position for this host candidate. We do not find any other objects near the XRT error circle down to the limiting magnitudes in Table 2 ,

\subsection{GRB 060223}

Berger et al. (2006) found a redshift of $z=$ 4.41 for GRB 060223 (Cummings et al. 2006), and only the reddest band $(V)$ of Swift-UVOT detected the OT of this high- $z$ event.

Two neighboring pixels inside the OT error circle (Cummings et al. 2006) are at the $3 \sigma$ level above the background (see Fig. 1), but we can obviously not claim detection of a source. We note that our limiting magnitude for this GRB field is $R=25.3$.

\subsection{GRB 060313}

This is the only short burst in our sample (Pagani et al. 2006a; Roming et al. 2006). The first afterglow report was by Levan et al. (2006). The first night's images contained the OT, so later observations were used to search for the host galaxy. Due to unfavorable seeing and moon illumination our $R$-band limit is only $23.9 \mathrm{mag}$, and no sources are seen in the XRT error circle.

\section{Comparison with pre-Swift GRB host sample}

Fruchter et al. (2006) have compiled a sample of 46 galaxies that hosted long GRBs with well-localized OT positions detected prior to the launch of the Swift satellite. Discarding two galaxies without a brightness estimate and the somewhat controversial and nearby $z=0.0085$ GRB 980425, this pre-Swift sample contains photometry in the AB photometric system for 43 GRB host galaxies see Fig. 3. The quoted AB magnitudes are a mix of several different filters, primarily HST "V-like" filters - F606W, F555W, and "CLEAR" - but also F775W (two cases) and F814W (one case). Our sample, i.e. the magnitudes from Table 2, can not be directly compared with the Fruchter et al. sample for several reasons: First, our sample contains several bursts without a confirmed OT. Second, 


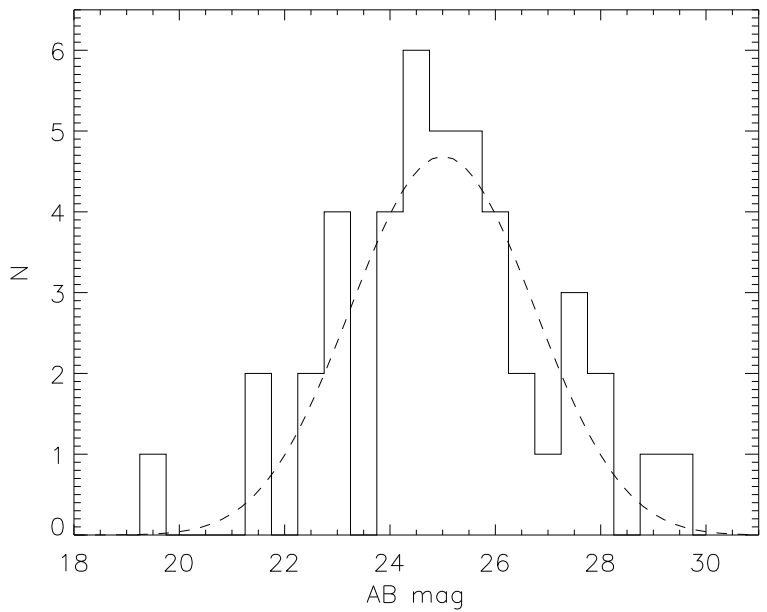

Fig. 3.- Histogram of the pre-Swift GRB host galaxy sample of Fruchter et al. (2006). Bin size $=0.5$ mag. The dotted line is a beta function fit to the data (see text).

our table lists $R$ - and $V$-magnitudes, not $\mathrm{AB}$ magnitudes, for the hosts and upper limits. Third, we detected a host galaxy candidate only for a few bursts. This last point renders several standard statistical methods for sample comparison (e.g. a direct KolmogorovSmirnov test) inapplicable. Instead we have applied the upper limits in the analysis and tested, using Monte Carlo simulations, how many GRB hosts we should have detected based on the distribution of host brightnesses from Fruchter et al. (2006). At this point we want to note that the following comparison between the two samples is not statistically robust, as neither our sample nor the preSwift sample of Fruchter et al. is complete. If we consider only long Swift GRBs with confirmed OTs, our data include $50 \%$ of all targets observable from La Silla during our two observing runs. Fruchter et al.'s sample (constrained by having OTs and being in the long burst category) is probably less complete, since only about a third of the detected preSwift GRBs are sufficiently well localized to permit host identifications (e.g., Fvnbo et al.
2001; Berger et al. 2002). We nevertheless choose to include this comparison as an exercise in order to check whether our observations are comparable with the host magnitude distribution in Fruchter et al. (2006)

Before we carried out the simulations we first defined a subset of the Danish $1.54 \mathrm{~m}$ observations which comprises 15 long GRBs detected with Swift and which have a confirmed optical transient: 050318, 050401, 050406, 050416, 050502B, 050603, 050607, 050726, 050801, 050826, 050908, 050915, 050922C, 051016B, and 060223. Short bursts, HETE bursts, and bursts without a confirmed OT were omitted. Next, since the Fruchter et al. sample quotes magnitudes in the $\mathrm{AB}$ system we transformed our $R$ and $V$ upper limits for the host galaxies into the AB system according to Fukugita et al. (1995). Finally, dust maps from Schlegel et al. (1998) were used to correct for foreground Galactic extinction. The AB magnitudes of both our and Fruchter et al.'s sample encompass bands from the visible to the red; this will widen the host magnitude distributions compared to samples observed with a single filter, but this fact should not seriously affect the conclusions we draw below.

The rationale for performing Monte Carlo simulations is that we want to see how many host galaxies we would have detected had the distribution of host magnitudes of our targets matched that of the Fruchter et al. sample. This will be compared to the four host detections we claim (concerning GRBs 050416, 050826, 050915, and 051016B).

We need to quantify the Fruchter et al. host magnitude distribution; this was done by fitting a beta distribution function 4 (which allows for skewness) to the magnitude histogram, see the dotted line in Fig. 3. Even

\footnotetext{
${ }^{4}$ Unnormalized beta distribution: $f(x ; \alpha, \beta) \sim$ $x^{\alpha-1}(1-x)^{\beta-1}$, with parameters $\alpha, \beta>0$
} 
though the sample contains 43 hosts, the sampling is rather sparse in the wings. We tried fitting different functions to the histogram, but this did not significantly change the end results of the analysis.

Using the beta probability density function as an approximation of the host magnitude distribution in Fruchter et al., one can draw magnitudes (using a random seed) and then compare them one by one to our 15 upper limits. If the magnitude drawn is less i.e. brighter - than the upper limit (transformed to the $\mathrm{AB}$ photometric system and corrected for foreground Galactic extinction, as described above) it is recorded as a host detection. Having done this for all the 15 upper limits one finally sums the number of detections. This procedure is repeated 10000 times, thus producing a distribution of the number of host detections, see Fig. 4. (We also checked that the simulated magnitudes based on the fitted beta distribution was comparable to the original Fruchter et al. sample. The mean of the simulated values was exactly the same as for the original sample, and the median and the scatter was also similar.)

While we found four host galaxies out of our 15 GRB subsample, the mean number of detected host galaxies from the simulations is 9.0, with a standard deviation of 1.8. In fact, 99.3\% of the Monte Carlo simulations yielded five or more host detections. This confidence level should not be taken at face value due to the statistical incompleteness of the samples. This coupled with the fact that the possible biases and selection effects in each of them are hard to quantify, makes a definite and quantitative conclusion impossible. We have in this exercise simply tried to constrain our observed GRB targets in the same way (solely long GRBs with confirmed OT) as in the Fruchter et al. sample and then performed Monte Carlo runs to investigate whether the two samples are comparable. The above simu-

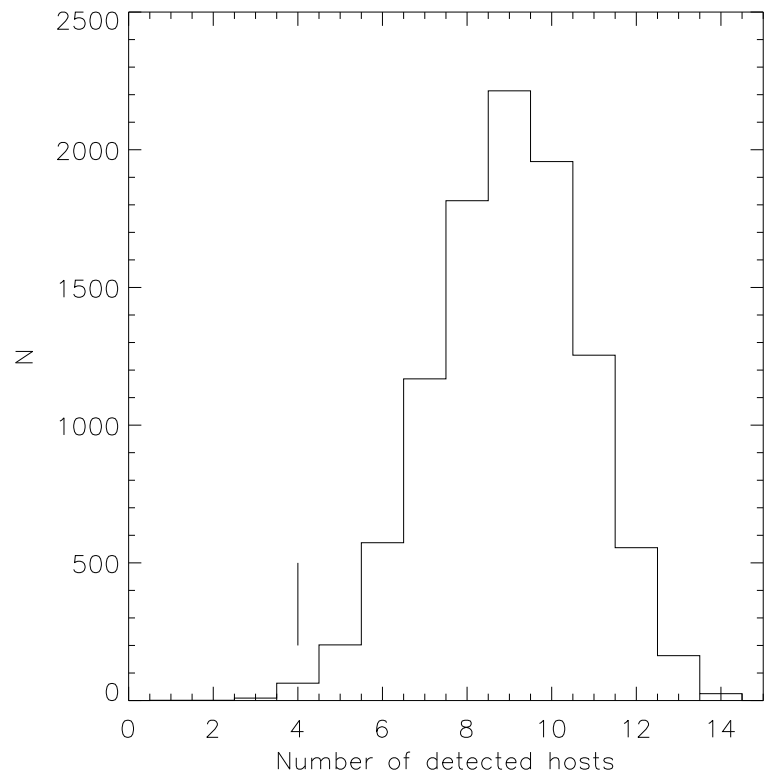

Fig. 4.- Histogram showing the distribution of host galaxy detections from the Monte Carlo simulations. We detected four hosts (indicated by the vertical line) from our 15 GRB fields, while the simulations indicate that we should have detected $9.0 \pm 1.8(1 \sigma)$ had the host magnitude distribution of our sample been similar to the one in Fruchter et al. (2006).

lations indicate that the Swift GRB host sample presented here is fainter than the pre-Swift host sample of Fruchter et al. While this can not be extended to hosts in general due to the incompleteness of both samples, we do consider it tentative evidence of fainter Swift hosts.

Concerning possible biases, we want to make a couple of comments. Since GRBs which were undetected in ground-based followups often were later chosen as targets for the HST programs on which the Fruchter et al. data is based, one could argue that this would bias the Fruchter et al. sample towards the faint end of the pre-Swift host luminosity function. If so, this would strengthen our findings. Secondly, large Galactic extinctions 
can not explain or contribute to the faintness of our GRB hosts, as only one field (i.e. GRB 050826, whose host is in fact detected) has significant foreground extinction.

To summarize, we detect four hosts in a subsample of 15 GRBs selected using comparable criteria to that of Fruchter et al. (2006). Assuming, for a moment, that the host magnitude distribution of Fruchter et al. is universal, we would expect to detect some 7-11 hosts given our observing conditions. If we had observed every Swift-detected GRB field observable from La Silla during our two runs we should have detected about twice as many hosts, i.e. about 18. To achieve this we would have to detect a host for just about every remaining unobserved GRB field. We can not find a plausible reason why our observed sample should be selected in such a heavily biased way. It seems reasonable that the most natural explanation for our observed lower-thanexpected rate of host detections is that the hosts are fainter than the Fruchter et al. sample.

\section{Summary}

The large observational data set of 24 GRB fields obtained with the Danish $1.54 \mathrm{~m}$ telescope on La Silla have been used to search for the galaxies hosting these bursts. New, previously unpublished, host galaxy candidates are presented for GRBs 050915 and 051021. We also suggest possible hosts for GRBs 050412, 050714B, 050826, 051006, and 051117B; these objects are reported in early GCN circulars as part of the search for the OT, but since we detect them months after the bursts they are not transient sources and, consequently, qualify as at least candidate hosts. Photometry for the host galaxies of GRBs 050416, 051016B, and 051022 is also provided. These galaxies already have measured redshifts from emission lines, but to our knowledge no previously published photometry. Magnitudes (not cor- rected for Galactic extinction) and positions are given in Table 2, as well as upper limits in various photometric bands for the nondetections.

We fail to detect more than half of the hosts in our GRB sample, despite rather deep limits. These fields, along with a couple of our marginal detections, e.g. GRBs 050915 and 051021, should be imaged with larger telescopes. Likewise, the new host candidates presented here are prime targets for spectroscopic follow-up to determine the redshift and probe extinction, star-formation rate etc. Redshift determinations for the hosts would also fix the energy and time scale of the bursts and afterglows, and thus contribute to the increasing data base and knowledge on the GRB phenomenon.

It is worth noting that as far as detected sources (not necessarily GRB hosts) inside or on the border of the Swift XRT error circle are concerned, they seem to be brighter (and apparently larger) for bursts which have no detected OT. From our sample, the median AB magnitude of sources inside the XRT error circle of such GRBs is 21.8, whilst the corresponding value for the galaxies hosting GRBs with optical afterglows is 23.6 mag. This is admittedly small number statistics, but it could be an indication that galaxies hosting GRBs with very faint or even missing OTs belong to a different population (i.e. more dusty galaxies with more homogeneously distributed star-formation) than galaxies hosting GRBs with optical afterglows. (We avoid referring to GRBs without a detected OT as "dark" bursts, as there are no generally accepted criteria for what constitutes an optically dark GRB.)

Our Monte Carlo simulations indicate that the host galaxy sample presented here (i.e. the 15 GRB fields corresponding to long, Swiftdetected, OT-confirmed GRBs) is fainter than the pre-Swift sample in Fruchter et al. (2006). 
A possible explanation for this is that they are more distant than the hosts in Fruchter et al. This is in accordance with the observation that Swift GRBs are at higher average redshift than pre-Swift bursts (e.g. Jakobsson et al. 2006a). Consequently, GRBs detected by the Swift satellite provide a better insight into the early history of the Universe. The faintness of the GRB host galaxies calls for more extensive observation efforts with large $8 \mathrm{~m}$ class or space telescopes in order to construct any representative sample of GRB host galaxies (e.g. Jakobsson et al. 2006b).

We are grateful to the staff at ESO/La Silla for help with the control system at the Danish $1.54 \mathrm{~m}$ telescope and to Brian Lindgren Jensen at the Dark Cosmology Centre for software support during the observing runs.

\section{REFERENCES}

Angelini, L., et al. 2005, GCN \#3161

Band, D., et al. 2005, GCN \#4281

Band, D. L., \& Hartmann, D. H. 1998, ApJ, 493, 555

Barthelmy, S., et al. 2005, GCN \#3682

Berger, E., \& Becker, G. 2005, GCN \#3520

Berger, E., et al. 2002, ApJ, 581, 981

Berger, E., et al. 2005, GCN \#3185

Berger, E., et al. 2006, GCN \#4815

Berger, E., \& McWilliam, A., et al. 2005, GCN \#3511

Berger, E., \& Mulchaey, J. 2005, GCN \#3122

Bertin, E., \& Arnouts, S. 1996, A\&AS, 117, 393

Blain, A. W., \& Natarajan, P. 2000, MNRAS, 312, L35
Bloom, J. S. 2005, GCN \#3990

Blustin, A.J., et al. 2005a, GCN \# 3733

Blustin, A.J., et al. 2005b, GCN \#3849

Bolzonella, M., Miralles, J.-M., \& Pelló, R. 2000, A\&A, 363, 476

Boyd, P., et al. 2005, GCN \#4096

Brown, P., et al. 2005, GCN \#3516

Butler, N. R. 2006, astro-ph/0611031

Cameron, P.B., et al. 2005, GCN \#3513

Cameron, P.B., \& Frail, D. A. 2005, GCN \#4154

Cenko, S.B., et al. 2005a, GCN \#3265

Cenko, S.B., et al. 2005b, GCN \#3358

Cenko, S.B., et al. 2005c, GCN \#3542

Chen, Y.T., et al. 2005a, GCN \#4105

Chen, Y.T., et al. 2005b, GCN \#4285

Christensen, L., Hjorth, J., \& Gorosabel, J. 2004, A\&A, 425, 913

Covino, S., et al. 2005, GCN \# 3616

Cummings, J.R, et al. 2005, GCN \#3237

Cummings, J.R., et al. 2006, GCN \#4813

de Pasquale, M., et al. 2005, GCN \#3123

de Pasquale, M., et al. 2006, MNRAS, 365 , 1031

de Ugarte, A., et al. 2005, GCN \#4064

Falcone, A. D., et al. 2005, GCN \#3330

Falcone, A. D., et al. 2006, ApJ, 641, 1010

Fenimore, E. E., et al. 1993, Nature, 366, 40

Fox, D. B., et al. 2005a, GCN \#3244 
Fox, D. B., et al. 2005b, GCN \#4120

Fruchter, A. S., et al. 2006, Nature, 441, 463

Fukugita, M., Shimasaku, K., \& Ichikawa, T. 1995, PASP, 107, 945

Fynbo, J.U., et al. 2001, A\&A, 369, 373

Fynbo, J.P.U., et al. 2005a, GCN \#3176

Fynbo, J.P.U., et al. 2005b, GCN \#3736

Gal-Yam, A., et al. 2005, GCN \#4156

Gehrels, N., et al. 2004, ApJ, 611, 1005

Goad, M., et al. 2005, GCN \#3942

Godet, O., et al. 2007, astro-ph/0702262

Grupe, D., et al. 2005, GCN \#3977

Grupe, D., et al. 2006, ApJ, 645, 464

Halpern, J.P., et al. 2005, GCN \#3891

Halpern, J.P., et al. 2006a, GCN \#4749

Halpern, J.P., et al. 2006b, GCN \#5982

Hogg, D. W., \& Fruchter, A. S. 1999, ApJ, 520,54

Holland, S.T, et al. 2007, AJ, 133, 122

Jakobsson, P., et al. 2005a, GCN \#4015

Jakobsson, P., et al. 2005b, GCN \#4029

Jakobsson, P., et al. 2006a, A\&A, 447, 897

Jakobsson, P., et al. 2006b, astro-ph/0611561

Jensen, B.L., et al. 2005, GCN \#3243

Kosugi, G., et al. 2005, GCN \#3263

Krimm, H., et al. 2005, GCN \#3111

Le Floc'h, E., et al. 2003, A\&A, 400, 499

Levan, A.J., et al. 2005, GCN \#3613
Levan, A.J., et al. 2006, GCN \#4871

Li, W., et al. 2005, GCN \#3945

Malesani, D., et al. 2005, GCN \# 3614

Mangano, V., et al. 2005, GCN \#3884

Mao, S., \& Mo, H. J. 1998, A\&A, 339, L1

McNaught, R., et al. 2005, GCN \#3163

Monet, D. G., et al. 2003, AJ, 125, 984

Mulchaey, J., \& Berger, E. 2005, GCN \#3114

Nakagawa, Y. E., et al. 2006, PASJ, 58, L35

Norris, J., et al. 2005a, GCN \#4013

Norris, J., et al. 2005b, GCN \#4061

Olive, J.-F., et al. 2005, GCN \#4131

Ovaldsen, J.-E., Teuber, J., Schild, R. E., \& Stabell, R. 2003a, A\&A, 402, 891

Ovaldsen, J.-E., Teuber, J., Stabell, R., \& Evans, A. K. D. 2003b, MNRAS, 345, 795

Pagani, C., et al. 2006a, GCN \#4867

Pagani, C., et al. 2006b, ApJ, 645, 1315

Parsons, A., et al. 2005a, GCN \#3180

Parsons, A., et al. 2005b, GCN \#4103

Poole, T., et al. 2005, GCN \#3698

Prochaska, J. X., et al. 2005, GCN \#3971

Retter, A., et al. 2005a, GCN \#3509

Retter, A., et al. 2005b, GCN \#3525

Rhoads, J., et al. 2005, GCN \#3527

Rich, J., et al. 2005 GCN \#3338

Rol, E., et al. 2005, GCN \#3186

Roming, P. W. A., et al. 2006, ApJ, 651, 985 
Rumyantsev, V., et al. 2005, GCN \#4159

Rykoff, E. S., et al. 2005, GCN \#3723

Rykoff, E. S., et al. 2006, ApJ, 638, L5

Sakamoto, T., et al. 2005, GCN \#3264

Schady, P., et al. 2005, GCN \#3276

Schlegel, D. J., Finkbeiner, D. P., \& Davis, M. 1998, ApJ, 500, 525

Soderberg, A.M., et al. 2006, astro-ph/0607511

Soderberg, A.M., et al. 2005a, GCN \#3187

Soderberg, A.M., et al. 2005b, GCN \#4186

Teuber, J. 1993, Digital image processing, Prentice Hall

Thöne, C., et al. 2005, GCN \#4291

Torii, K., 2005, GCN \#3943

Totani, T. 1997, ApJ, 486, L71

Watson, D., et al. 2006, ApJ, 652, 1011

Wijers, R. A. M. J., Bloom, J. S., Bagla, J. S., \& Natarajan, P. 1998, MNRAS, 294, L13

Yoshida, A., et al. 2005, GCN \#4116

This 2-column preprint was prepared with the AAS LATEX macros v5.2. 